\title{
Identification and differential expression of piRNAs in the gonads of Amur sturgeon (Acipenser schrenckii)
}

\author{
Lihong Yuan ${ }^{\text {Corresp., }}{ }^{1,2}$, Linmiao Li ${ }^{2}$, Xiujuan Zhang ${ }^{2}$, Haiying Jiang ${ }^{2}$, Jinping Chen Corresp. 2 \\ 1 School of Life sciences and Biopharmaceutics, Guangdong Pharmaceutical University, Guangzhou, China \\ 2 Guangdong Key Laboratory of Animal Conservation and Resource Utilization, Guangdong Public Laboratory of Wild Animal Conservation and Utilization, \\ Guangdong Institute of Applied Biological Resources, Guangzhou, China \\ Corresponding Authors: Lihong Yuan, Jinping Chen \\ Email address: ylh@gdpu.edu.cn, chenjp@giabr.gd.cn
}

Objective: Sturgeons are considered living fossils, and have a very high conservation and economic value. Studies on the molecular mechanism of sturgeon gonadal development and sex differentiation would not only aid in understanding vertebrate sex determination but also benefit sturgeon aquaculture. Piwi-interacting RNAs (piRNAs) have been shown to function in germline or gonadal development. In this study, we performed small RNA deep sequencing and microarray hybridization to identify potential sturgeon piRNAs. Methods: Male and female sturgeon gonads were collected and used for small RNA sequencing on an Illumina HiSeq platform with the validation of piRNA expression by microarray chip. The program Bowtie and $k$-mer scheme were performed to filter small RNA reads and discover potential sturgeon piRNAs. A known piRNA database, the coding sequence (CDS), 5' and 3' untranslated region (UTR) database of the A. Schrenckii transcriptome, Gene Ontology (GO) database and KEGG pathway database were searched subsequently to analyze the potential bio-function of sturgeon piRNAs. Results: A total of 875,679 putative sturgeon piRNAs were obtained, including 93 homologous to known piRNAs and hundreds showing sex-specific and sex-biased expression. Further analysis showed that they are predominant in both the ovaries and testes and those with a sex-specific expression pattern are nearly equally distribution between sexes. This may imply a relevant role in sturgeon gonadal development. KEGG pathway and GO annotation analyses indicated that they may be related to sturgeon reproductive processes. Conclusion: Our study provides the first insights into the gonadal piRNAs in a sturgeon species and should serve as a useful resource for further elucidation of the gene regulation involved in the sex differentiation of vertebrates. These results should also facilitate the technological development of early sex identification in sturgeon aquaculture. 


\section{Identification and differential expression of piRNAs in the gonads of}

\section{Amur sturgeon (Acipenser schrenckii)}

3

4

5 Lihong Yuan ${ }^{1,2}$, Linmiao Li ${ }^{2}$, Xiujuan Zhang², Haiying Jiang ${ }^{2}$, Jinping Chen ${ }^{2}$

6

7

8 1. School of Life science and Biopharmaceutics, Guangdong Pharmaceutical University,

$9 \quad$ Guangzhou, China

10 2. Guangdong Key Laboratory of Animal Conservation and Resource Utilization, Guangdong

11 Public Laboratory of Wild Animal Conservation and Utilization, Guangdong Institute of

12 Applied Biological Resources, Guangzhou, China

13

14 * Corresponding author:

\section{Lihong Yuan}

16 Email: ylh@gdpu.edu.cn

\section{Jinping Chen}

18 Email: chenjp@giabr.gd.cn 


\section{Abstract}

27 Objective: Sturgeons are considered living fossils, and have a very high conservation and

28 economic value. Studies on the molecular mechanism of sturgeon gonadal development and sex

29 differentiation would not only aid in understanding vertebrate sex determination but also benefit

30 sturgeon aquaculture. Piwi-interacting RNAs (piRNAs) have been shown to function in germline

31 or gonadal development. In this study, we performed small RNA deep sequencing and

32 microarray hybridization to identify potential sturgeon piRNAs.

33 Methods: Male and female sturgeon gonads were collected and used for small RNA sequencing

34 on an Illumina HiSeq platform with the validation of piRNA expression by microarray chip. The

35 program Bowtie and $k$-mer scheme were performed to filter small RNA reads and discover 36 potential sturgeon piRNAs. A known piRNA database, the coding sequence (CDS), 5' and 3'

37 untranslated region (UTR) database of the A. Schrenckii transcriptome, Gene Ontology (GO) 
38 database and KEGG pathway database were searched subsequently to analyze the potential bio-

39 function of sturgeon piRNAs.

40 Results: A total of 875,679 putative sturgeon piRNAs were obtained, including 93 homologous

41 to known piRNAs and hundreds showing sex-specific and sex-biased expression. Further

42 analysis showed that they are predominant in both the ovaries and testes and those with a sex-

43 specific expression pattern are nearly equally distribution between sexes. This may imply a

44 relevant role in sturgeon gonadal development. KEGG pathway and GO annotation analyses

45 indicated that they may be related to sturgeon reproductive processes.

46 Conclusion: Our study provides the first insights into the gonadal piRNAs in a sturgeon species

47 and should serve as a useful resource for further elucidation of the gene regulation involved in

48 the sex differentiation of vertebrates. These results should also facilitate the technological

49 development of early sex identification in sturgeon aquaculture.

\section{INTRODUCTION}

52 Sturgeons (order: Acipenseriformes, infraclass: Chondrostei) are referred to as living fossils and

53 have considerable value in aquaculture as sturgeon eggs (caviar) (Bemis et al., 1997; Honkala

54 and Lobstein, 2010). Sturgeons contain 25 caviar-producing species, 17 of which are members of

55 the Acipenser genus (Bemis et al., 1997). Due to the huge profits associated with the sale of

56 sturgeon caviar, over-exploitation of wild stocks occurred worldwide throughout the $20^{\text {th }}$ century, 
57 and major sturgeon fisheries are in decline (Pikitch et al., 2005). Currently, all Acipenser species

58 appear on the IUCN Red List of Critically Endangered Species (Ruban, 2013) and under

59 Appendices I or II of CITES (Convention on International Trade in Endangered Species)(Dongol,

60 2011). Given the decline of wild stocks and conservation, great efforts have been made to

61 develop commercial sturgeon aquaculture to meet the demand for caviar, originally in Europe

62 and North America but more recently in Russia, Iran and China (Raymakers and Hoover, 2010;

63 Wei et al., 2011). Currently, it is estimated that approximately $50 \%$ of the caviar in trade is from

64 farmed stocks (Bronzi et al., 2011).

66 The rapid development of sturgeon aquaculture has greatly decreased the pressure on wild stocks;

67 however, aquaculture brings new challenges. Two of the primary challenges are performing early

68 sex identification and culling individuals with gonad dysplasia. Due to the long culture period in

69 sturgeon (with an estimated 15 years/generation), approximately $50 \%$ of the offspring are

70 profitless males, and up to $30 \%$ of offspring have gonad dysplasia (Krykhtin and Svirskii, 1997).

71 Performing early sex identification, increasing the proportion of female sturgeon and decreasing

72 gonad dysplasia may significantly improve sturgeon farming profits. Currently, the methods used

73 in sturgeon sex and reproductive stage determination (i.e., laparoscopy, ultrasonography,

74 histology and sex steroid analyses (Falahatkar et al., 2011; Petochi et al., 2011)) are highly

75 dependent on technician experience and are restricted by age and gonad maturity (Devlin and 
76 Nagahama, 2002; Masoudifard et al., 2011). Therefore, a better understanding of the processes

77 that regulate sexual development, especially gonadogenesis and gametogenesis, may provide

78 novel targets in sturgeon aquaculture. Furthermore, in contrast to mammals, the sex of lower

79 bony fish is unstable and may be affected by many factors such as the environment and

80 hormones (Devlin and Nagahama, 2002). However, due to the complexity of the number and

81 ploidy of their chromosomes and lack of knowledge regarding sex chromosome differentiation,

82 the study of sex determination and differentiation in sturgeons at the molecular level is still

83 difficult.

85 Piwi-interacting RNAs (piRNAs), a distinct class of 26-32 nt non-coding RNAs, have been

86 shown to function in germline development, transposon silencing and epigenetic regulation

87 mediated by Piwi proteins (Ashe et al., 2012; Houwing et al., 2008; Juliano et al., 2011; Malone

88 and Hannon, 2009; Ross et al., 2014; Vagin VV, 2006). Recently the piRNA biology has been

89 expanded rapidly in not only embryonic patterning, germ cell specification, but also in stem cell

90 biology, neuronal activity and metabolism (Rojas-Ríos et al., 2018). Studies have shown that

91 piRNAs are expressed in both the male and female germlines of Caenorhabditis elegans,

92 Drosophila melanogaster, Danio rerio and Xenopus laevis and in the male germlines of

93 mammals and birds but have limited expression in the early female germlines of mammals (Ashe

94 et al., 2012; Ha et al., 2014; Houwing et al., 2008; Juliano et al., 2011; Lau et al., 2009; Li et al., 
95 2013; Qingling Yang, 2013; Roovers et al., 2015; Wilczynska et al., 2009; Williams et al., 2015).

96 A deficiency in the genes required for piRNA biogenesis affects the regulation of gene silencing,

97 cell differentiation and gonadal development in animals. However, the distribution of piRNAs in

98 sturgeon gonads and the function in sex differentiation remains unclear.

Acipenser schrenckii (Amur sturgeon) is an economically important sturgeon species in China,

101 and the wild stocks are mainly distributed in the Amur River, Songhua River and Heilong River

102 (Li et al., 2012). Sturgeon small RNA transcriptome and gene expression patterns in many

103 tissues (including the gonads) have been assayed using RNA-Seq technology, and a batch of sex-

104 biased RNAs have been identified (Jin et al., 2015; Yuan et al., 2014; Zhang et al., 2016a; Zhang

105 et al., 2016b). These studies support sturgeon gonad development research. In the present study,

106 we first analyzed the putative piRNAs of Amur sturgeon gonads using the Illumina sequencing

107 platform. We combined these data with the results of microarray expression validation to identify

108 the putative sturgeon-specific and/or sex-specific piRNAs and illustrate the potential role of

109 putative piRNAs on sturgeon gonadal development and sex differentiation. This study should

110 provide information regarding piRNAs in gonads, help to reveal the mechanism of the sex

111 duality of sturgeon, and contribute to identifying gender-related bio-markers for use in sturgeon 112 aquaculture. 


\section{MATERIALS AND METHODS}

\section{Ethics statement}

116 The protocol was approved by the Committee on the Ethics of Animal Experiments of

117 Guangdong Institute of Applied Biological Resources (GIABR2014008). Individual sturgeon

118 were immersed in water with 10-4 (v/v) eugenol for approximately 1-3 minutes for euthanasia,

119 according to the AVMA guidelines (Leary et al., 2013). All efforts were made to minimize

120 suffering.

121

\section{Sample and RNA preparation}

123 In this study, we used 3-year-old Amur sturgeons (Acipenser schrenckii), whose sex could be

124 identified accurately by laparoscopy and histology. The animals were obtained from the

125 Engineering and Technology Center of Sturgeon Breeding and Cultivation of the Chinese

126 Academy of Fishery Science (Beijing, China). The testes and ovaries of six 3-year-old Amur

127 sturgeons (three males and three females) were collected. Total RNA was extracted from tissue

128 samples separately with RNAiso reagent (TaKaRa, Japan) according to the manufacturer's

129 instructions. The RNA concentrations were measured using a Qubit RNA Assay Kit in Qubit 2.0

130 Fluorometer (Life Technologies), and RNA purity was assessed using a Nano Photometer

131 spectrophotometer (IMPLEN). RNA integrity was inspected using an RNA Nano 6000 Assay

132 Kit and Bioanalyzer 2100 system (Agilent Technologies). 


\section{Small RNA library preparation and sequencing}

135 Four RNA samples ( 2 testes and 2 ovaries, $3 \mu \mathrm{g}$ RNA of each) were used for the construction

136 and sequencing of small RNA libraries. In brief, NEB 3' SR Adaptor ligation, SR RT Primer

137 hybridization and NEB 5' SR Adaptor ligation were performed according to the NEBNext

138 Multiplex Small RNA Sample Preparation Set (Illumina) protocol. After first strand cDNA

139 synthesis using M-MuLVA Reverse Transcriptase (RNase $\mathrm{H}^{-}$) by PCR, the 140 bp to 160 bp

140 products (with adaptors on both sides) were separated on an 8\% PAGE gel and quantified using

141 an Agilent Bioanalyzer 2100 system. Then, a cluster of index-coded samples was generated

142 using a TruSeq SR Cluster Kit v3-cBot-HS (Illumina) and sequenced on an Illumina HiSeq 2000

143 platform. Finally, 50 bp single-end reads were generated.

\section{Small RNA annotation and piRNA identification}

146 In this study, the testis and ovary transcriptomes of A. Schrenckii (Jin et al., 2015) were used as

147 reference sequences for small RNA annotation. After removal of unclean reads (adapters, low

148 quality reads, reads containing 'n', and redundant reads), clean unique reads were mapped onto

149 the $A$. schrenckii transcriptome reference sequences using the program Bowtie (Langmead and

150 Pop, 2009) with no mismatches allowed. Perfectly mapped reads were filtered by 3 successive

151 steps to remove small RNA elements: 1) searched against Sanger miRBase (Release 19) to 
152 exclude conserved miRNAs; 2) screened against Rfam (http://rfam.sanger.ac.uk/) and

153 RepeatMasker (http://www.repeatmasker.org/) with Bowtie to filter the sequences originating

154 from rRNA, tRNA, snRNA, snoRNA and repeats; 3) analyzed by miREvo, mirdeep2 and

155 MirCheck to remove the potential novel miRNA reads. The detailed process of small RNA filter

156 was described in our previous study (Yuan et al., 2014).

158 The remaining reads with lengths of 26-32 nt were used for piRNA discovery. A $k$-mer scheme

159 relied on the training sets of non-piRNA and the piRNA sequences of five model species (rat,

160 mouse, human, fruit fly and nematode), was applied as previously described (Zhang et al., 2011).

161 Then, it was compared with the existing 'static' scheme on the basis of the position-specific base

162 usage. Putative novel piRNAs were scanned against piRNABank, (http://pirnabank.ibab.ac.in)

163 using Bowtie with no mismatches allowed to identify the orthologs of known piRNAs. The

164 relative frequencies of piRNA nucleotide utility reads were analyzed. Subsequently, the putative

165 piRNAs were functionally annotated using the coding sequence (CDS), 5' and 3' untranslated

166 region (UTR) database of the A. Schrenckii transcriptome with TransDecoder software

167 (https://github.com/trinityrnaseq/trinityrnaseq/wiki/Coding-Region-Identification-in-Trinity-

168 Assemblies).

169

170 piRNA microarray and data analysis 
171 To validate the expression of putative piRNAs identified by Illumina sequencing, we selected: 1)

1721,092 that exhibited significantly different expression in ovaries vs. testes with $|\operatorname{logFC}| \geq 1.5, \mathrm{P}$

$173 \leq 0.01$, Padj $\leq 0.05$, and read counts $>10$; and 2) 779 highly expressed putative piRNAs with

174 read counts $50 \sim 4,646$ in ovaries or $50 \sim 8177$ in testes. For each of the six RNA samples (three

175 ovaries and three testes, extracted above) assessed, $4 \mu \mathrm{g}$ of total RNA were used to hybridize

176 with the microarray chip.

177

178 A piRNA microarray was manufactured by RIBOBIO (China), and each piRNA probe had three

179 replicates. The chip was hybridized with single-color labeling (Cy5) RNAs according to the

180 manufacturer's protocol, with no modifications. Microarray results were extracted using a laser

181 scanner (GenePix 4000B, Molecular Device) and digitized using Array-Pro image analysis

182 software (Media Cybernetics). Raw data were subtracted using the background matrix, and spots

183 with $\mathrm{CV}[($ standard deviation $) /($ signal intensity $)]<0.3$ were normalized using a quantile

184 normalization method to remove system related variations, including sample amount variations

185 and signal gain differences of the scanners and to faithfully reveal the biological variations

186 (Bullard et al., 2010). The medians of repeated data (normalized intensity) were used for

187 statistical analyses with One-Way ANOVA method, and the expression of piRNAs was deemed

188 significant with the criterion $|\log \mathrm{FC}| \geq 1$ and $\mathrm{P} \leq 0.05$. 
191 Putative piRNAs were mapped onto the A. schrenckii transcriptome using the program Bowtie

192 with no mismatches allowed to identify piRNA-generating genes. The piRNA-generating genes

193 were mapped to Gene Ontology (GO) database (http://www.geneontology.org/) by Interproscan

194 (Zdobnov et al., 2001) and KEGG pathway database (http://www.genome.jp/kegg/) by BLASTX

195 at E values 1e-10 (Kanehisa et al., 2016), respectively by two levels, 1) total of piRNA-

196 generating genes and 2) sex-specific expression (only expressed in testes or ovaries and with a

197 read count $\geq 10$ ) or sex-biased expression of piRNA-generating genes in ovaries and testes

198 ( $|\log \mathrm{FC}| \geq 1$ and $\mathrm{P} \leq 0.05$ obtained by microarray). Finally, the enriched functional groups or 199 pathways were obtained with corrected $P<0.05$.

\section{RESULTS}

\section{Sequencing and statistics of small RNA reads}

A total of $7.7 \times 10^{6}-8.6 \times 10^{6}$ reads were sequenced from four small RNA libraries, with an error rate of $0.01 \%, \mathrm{Q} 30>97.3 \%$ and GC content of $\sim 48 \%$ (Table S1). Then, approximately 7.3 $\times 10^{6}-8.1 \times 10^{6}$ high-quality small RNA reads $(>94 \%$ in each library) were obtained after removal of ambiguous reads (Table S1). The size distribution and frequency percentage of small RNA reads are shown in Figure S1 and, of these, the potential piRNA reads (26 - $30 \mathrm{nt})$ were the 
209 schrenckii transcriptome reference sequences (Jin et al., 2015) were with a perfect match (Table

$210 \mathrm{~S} 1)$. A total of $3.5-6.7 \times 10^{4}$ reads were mapped to at least one putative Amur sturgeon miRNA

211 precursor by searching against miRBase, corresponding to $1.0-2.2 \%$ perfectly matched small

212 RNA reads (Table 1). Subsequent small RNA filtering indicated that other non-coding RNAs

213 (rRNA, tRNA, snRNA and snoRNA) and repeat sequences were approximately $1.0-1.5 \%$ and

$2140.3-0.4 \%$, respectively. Then, $5.3-6.6 \times 10^{3}(0.2 \%)$ potential novel miRNA reads specific to $A$.

215 schrenckii were detected with miREvo (Table 1).

216

217 piRNA discovery

218 According to a $k$-mer scheme analysis which relied on the training sets of non-piRNA and the

219 piRNA sequences of five model species (Zhang et al., 2011), we identified total of $5.7-6.2 \times 10^{5}$

220 piRNA reads in our sequencing results, that account for $16.6-19.8 \%$ of perfectly matched small

221 RNA reads (Table 1). By following steps described in the methods section, 875,679 putative

222 piRNAs from the testis and ovary libraries were predicted, including 93 piRNAs that were

223 homologous to known piRNAs (4 in fruit fly, 45 in zebra fish, 34 in human, 4 in mouse and 6 in

224 rat, see Table S2). Sturgeon putative piRNAs have a strong U bias at the extreme 5' position and

225 are enriched for adenine (A) at position 10 (Figure 1A). Further analysis indicated that their rate

226 of sex-specific expression (only expressed in ovary or testis) was up to $87 \%(767,805 / 875,679)$,

227 and the ratio of ovary- vs. testis-specific putative piRNAs was nearly 1:1 
$228(43.99 \%, 385,222 / 875,679$ vs. 43.69\%, 382,583/875,679, see Figure 1B). Moreover, we found a

229 similar percentage of sense and antisense strand putative piRNAs $(42.99 \%, 376,487 / 875,679$ vs.

$23057.01 \%, 499,192 / 875,679$, respectively). In total, approximately $42 \%(175,648$ in ovaries and

231 195,552 in testes) of all putative piRNAs were derived from well-annotated gene regions, 58\%

232 were from unannotated genomic regions (Figure 1C). Of these, only 31,949 (3.65\%) and 73,802

$233(8.43 \%)$ matched the 5' UTR and 3' UTR, respectively. Abundant piRNAs in gene regions were

234 found in CDS, which represented 265,499 (30.32\%) putative piRNAs.

235

236 Sturgeon putative piRNAs are present in both male and female gonads

237 Small RNA reads of both male and female sturgeon gonads displayed a peak in reads of 26-30 nt

238 in length (55.8\% in testes and 56.5\% in ovaries, shown in Figure 2A). This is an expected length

239 distribution for potential novel piRNA reads. Whereas Small RNA reads in 5 other somatic/non-

240 germline tissues (pool of brain, heart, muscle, liver and spleen tissues of $A$. schrenckii (Yuan et

241 al., 2014) showed a peak in reads of 20-24 nt in length which is an expected length distribution

242 for miRNA reads ( 52.9.4\%). Moreover, both ovary and testis samples had a strong 5' U bias in

243 reads of 20-24 nt (miRNA reads) and 26-30 nt (piRNAs reads) in length, whereas only reads of

244 20-24 nt among somatic samples had a 5' U bias (Figure 2B-2D). The library size of testis and

245 ovary is nearly equal (the ratio of total read counts $\approx 1: 1$, Table $\mathrm{S} 1$ ), thus we further compared

246 piRNAs vs miRNAs in sturgeon testis and ovary samples, and obtained a similar ratio of 
247 4.7:1 5.5:1 (Table 1). This clearly indicates putative piRNAs are far more abundant class of

248 small RNAs in both testis and ovaries compared to miRNAs. Moreover, both piRNA (1:1) and

249 miRNA (1.2:1) showed an equal ratio in testes vs. ovaries (Table 1).

250

251 piRNA-generating genes

252 A total of 875,679 putative piRNAs were mapped onto the A. schrenckii transcriptome

253 (including 122,381 unigenes in testis and 114,527 in ovaries, Jin et al., 2015). Total 49,390

254 piRNA-generating genes were obtained (23,079 in ovaries, 26,311 in testes, and 9634 in both).

255 The mean length of piRNA-generated genes was 967 nt (median 506), with a range of 150-

$25616,256 \mathrm{nt}$. There was a mean of 18 unique piRNAs reads per gene (scale of 1 up to 4,181

257 piRNAs per gene).

258

259 To illustrate the functions of the sturgeon putative piRNAs, gene function of the piRNA-

260 generated genes was annotated by searching against GO database with Interproscan and KEGG

261 pathway database by BLASTX at E values 1e-10. The GO analysis showed 264 enriched GO

262 terms for biological process, molecular function and cellular component, in them, $13 \mathrm{GO}$ terms

263 related to reproduction (such as sexual reproduction, mating/reproductive behavior, post-mating

264 regulation of female receptivity) were obtained (Table S3). Moreover, 29 enriched KEGG

265 pathways, including 6 involved in aquaculture, such as pathogen infection, multiple diseases, and 
antigen processing, were identified (Table S3). We further investigated the function of piRNA-

267 generated genes which with sex-specific piRNAs located (1,078 only detected in testis and 1,237

268 only detected in ovary, with read counts $\geq 10$ ), and more GO terms related to reproductive

269 processes were identified (Figure 3 and Table S4).

270

271 Validation of putative piRNA expression by microarray

272 We used an independent microarray platform to validate the expression of 1,871 putative

273 piRNAs (1,092 differently expressed and 779 highly expressed piRNAs, denoted ASY-piRNA-X,

274 where Xs are numerals) identified by Illumina sequencing. A total of 311 showed significantly

275 biased expression between sexes (deemed sex-biased expression) and were clustered into ten

276 clades including five clades up-regulated in ovaries and five clades up-regulated in testes (Figure

277 S2 and Table S5). Of these, 124 were specifically up-regulated in ovaries with $1<|\operatorname{logFC}|<8.5$

278 and $\mathrm{P} \leq 0.05$, and 187 were specifically up-regulated in testes with $1<|\operatorname{logFC}|<4.1$ and $\mathrm{P} \leq 0.05$

279 (Table S5). In sturgeon testes, the putative piRNA with the highest expression level was ASY-

280 piR-1255 with median of normalized intensity 4.04 in testes vs. 0.72 in ovaries (Table S5). In

281 contrast, the most expressed in the ovaries was ASY-piR-122 with median of normalized

282 intensity 13.33 in ovaries vs. 4.91 in testes (Table S5). Moreover, 1,335 were co-expressed in

283 both the testes and ovaries (median $>1$ in both and $\mathrm{P}>0.05$ ). Further analysis indicated no

284 significant difference in size distribution among sex-biased piRNAs (average $27.6 \mathrm{nt}$ in testes 
285 and $27.8 \mathrm{nt}$ in ovaries) and co-expressed piRNAs in both gonads (average $27.5 \mathrm{nt}$ ). The Illumina

286 sequencing and microarray expression of putative piRNAs was especially correlated for 311 sex-

287 biased piRNAs $(r=0.489, P=0.000)$. GO and KEGG annotation indicated that the 311 sex-

288 biased expressed piRNAs mainly participate in the metabolic processes of sturgeon gonads.

\section{DISCUSSION}

291 Here, we report the first identification of putative piRNAs and their expression in male and

292 female sturgeon (Acipenser schrenckii) gonads, thus greatly increasing the knowledge of

293 piRNAs in vertebrate gonadal development and/or sex differentiation. We obtained a total of

294875,679 putative piRNAs from sturgeon gonads by Illumina sequencing, and hundreds of them

295 showed sex-specific (only expressed in sturgeon testis or ovary) and sex-biased expressed

296 validated by microarray. In addition to basic physiological processes, many reproductive

297 processes (such as sexual reproduction, mating/reproductive behavior, post-mating regulation of

298 female receptivity) as well as pathogen infection and antigen processing are shown associated

299 with the expression of sturgeon putative piRNAs.

301 The development of an ultrahigh-throughput sequencing technique (RNA-seq) has allowed

302 researchers to discover and analyze the piRNAs of many organisms, and tens of thousands of 303 unique sequences have been identified (Brennecke et al., 2007; Castellano et al., 2015; Houwing 
304 et al., 2007; Williams et al., 2015). In this study, we obtained a total of $7.3 \times 10^{6}-8.1 \times 10^{6}$

305 high-quality small RNA reads, $58 \%$ of which were potential piRNA reads of 26-30 nt in length

306 (Table S1 and Figure S1). After mapping the reads to the $A$. schrenckii transcriptome reference

307 sequences, approximately $1.2-2.3 \%$ reads and $16.6-19.8 \%$ were identified as potential

308 miRNAs and piRNAs, respectively, whereas $>75 \%$ of the samples were unannotated genomic

309 regions (Table 1). By gathering additional sturgeon genomic and transcriptomic data and

310 comparing against the known sturgeon datasets, the accuracy of sturgeon gene annotation will

311 increase, and much more detailed information on $A$. schrenckii small RNAs can be uncovered

312 from the transcriptome datasets obtained in the present study.

313

314 Our study also revealed a large number of sturgeon-specific piRNAs and provided candidates for

315 further study of sturgeon gonadal development. A total of 875,679 sturgeon putative piRNAs

316 were identified, including 93 homologous to known piRNAs. The number of sturgeon putative

317 piRNAs were 10-fold and 40-fold higher than that in zebrafish ovaries and testes (Houwing et al.,

318 2007) and normal human testes (Yang Q, 2013), respectively. The large number of putative

319 piRNAs obtained in this study was probably due to chromosome ploidy resulting from multiple

320 and independent duplication events (Fontana et al., 2008; Ludwig et al., 2001). Approximately

$32186 \%$ of reads were sequenced only once, which may have been a result of the absence of

322 genomic information and the incomplete transcriptome annotation for sturgeons. 
324 Further analyses showed that the preference of 5' $\mathrm{U}$ and an adenine at position 10 (10A bias) in

325 sturgeon gonads is consistent with a known piRNA biogenesis by Piwi-mediated cleavage

326 (Brennecke et al., 2007) (Figure 1A). Moreover, Small RNA reads with characteristics typical to

327 piRNAs were found in both male and female sturgeon gonads, but not in somatic tissues (Figure

328 2A). We show that both expression of sex-specific and distribution on gene regions of putative

329 piRNAs are equally between testis and ovary (Figure 1B and 1C). The prevailing expression of

330 putative piRNAs in gonads has also been found in many other organisms (Ashe et al., 2012;

331 Houwing et al., 2007; Juliano et al., 2011; Lau et al., 2009; Wilczynska et al., 2009; Williams et

332 al., 2015) and provides insight into the possible roles of sturgeon piRNAs in gametogenesis

333 between sexes. Similar to the zebrafish, sturgeon putative piRNAs and miRNAs have equivalent

334 expression in the testes and ovaries (Table 1), indicating the important role of piRNAs and

335 miRNAs in the development of sturgeon gonads (Houwing et al., 2007). Putative piRNAs exhibit

336 significantly higher expression than putative miRNAs in both male and female sturgeon gonads

337 (Table 1), as has also been observed in both male and female zebrafish gonads, in normal adult

338 human testes and mouse testes (Aravin et al., 2006; Beyret et al., 2012; Girard et al., 2006;

339 Houwing et al., 2007; Yang Q, 2013). In addition, over 87\% of piRNAs showed sex-specific

340 expression; in contrast, only approximately 30\% of putative miRNAs (223/730) showed sex-

341 specific expression (Zhang et al, 2018). The predominant and equivalent male and female

342 sturgeon gonads expression of putative piRNAs suggest the key role of piRNAs (rather than 
343 miRNAs) in sturgeon gonad development and sex differentiation by acting through piRNA-Piwi

344 protein compound pathways.

345 To overcome the drawbacks of RNA-seq (Illumina sequencin), such as the potentiality of

346 sequencing errors and be influenced by raw data processing before small RNA identification (Git

347 et al., 2010), the expression of 1,871 putative piRNAs was validated by an independent

348 microarray platform. Data showed significantly sex-biased expression, including 124 that were

349 specifically highly expressed in ovaries and 187 in testes (Figure S2 and Table S5). There were

350 no significant differences in the size of sex-biased sturgeon putative piRNAs. A previous study

351 has shown that hsa-piR-020485 and hsa-piR-019825 (orthologs of ASY-piR-342 and ASY-piR-

352 1706) are significantly associated with sex (Yuan et al., 2016), a result consistent with our

353 observation of ASY-piR-342 and ASY-piR-1706 up-regulation in sturgeon ovaries (Table S5).

354 Moreover, we found that 1,335 putative piRNAs were co-expressed in male and female sturgeon

355 gonads (including 4 orthologs of known piRNAs), thus suggesting the crucial role of piRNAs in

356 sturgeon physiological processes. The identification of sex-biased piRNAs combined with the

357 co-expressed piRNAs in male and female gonads provides further insight into the molecular

358 mechanisms of sturgeon gonadal development and sex differentiation.

360 Annotation of piRNA-generating genes suggests that putative piRNAs are involved in multiple

361 reproductive processes (i.e., sexual reproduction, mating/reproductive behavior, and post-mating 
362 regulation of female receptivity). Moreover, 29 enriched KEGG pathways, including those

363 relates to common challenges in aquaculture, such as pathogen infection, multiple diseases, and

364 antigen processing, were identified (Table S3). Further enrichment analysis indicated that they

365 probably participate in reproduction, mainly via sex-specific expression (Figure 3 and Table S4).

\section{CONCLUSION}

368 This study greatly increase the knowledge of small regulatory RNAs in the sturgeon $A$.

369 Schrenckii. We have identified a large number of potential novel piRNAs and provided the first

370 description of the presence of piRNAs with likely roles in sturgeon gonadal development and sex

371 differentiation. Our data demonstrate that sturgeon putative piRNAs, similar to those of zebrafish,

372 are predominantly expressed in both male and female sturgeon gonads, thus supporting a

373 potentially conserved molecular function for piRNAs in sturgeon gametogenesis between sexes.

374 Moreover, the sex-specific expression of putative piRNAs suggests that they function in sturgeon

375 gonad development and sex differentiation. Furthermore, these gender-related piRNAs are the

376 candidates to be developed as DNA bio-marker for early sex-determination with less damaging

377 to sturgeon. Finally, information uncovered by this study aids in understanding sex determination

378 in vertebrates. 


\section{REFERENCES:}

382 Aravin, A., Gaidatzis, D., Pfeffer, S., Lagos-Quintana, M., Landgraf, P., Iovino, N., Morris, P., Brownstein,

383 M.J., Kuramochi-Miyagawa, S., Nakano, T., 2006. A novel class of small RNAs bind to MILI protein in 384 mouse testes. Nature 442, 203.

385 Ashe, A., Sapetschnig, A., Weick, E.M., Mitchell, J., Bagijn, M.P., Cording, A.C., Doebley, A.L., Goldstein, 386 L.D., Lehrbach, N.J., Pen, J.L., 2012. piRNAs Can Trigger a Multigenerational Epigenetic Memory in the 387 Germline of C. elegans. Cell 150, 88-99.

388 Bemis, W.E., Findeis, E.K., Grande, L., 1997. An overview of Acipenseriformes. Environmental Biology of 389 Fishes 48, 25-71.

390 Beyret, E., Liu, N., Lin, H., 2012. piRNA biogenesis during adult spermatogenesis in mice is independent of 391 the ping-pong mechanism. Cell Research 22, 1429-1439.

392 Brennecke, J., Aravin, A.A., Stark, A., Dus, M., Kellis, M., Sachidanandam, R., Hannon, G.J., 2007. Discrete 393 Small RNA-Generating Loci as Master Regulators of Transposon Activity in Drosophila. Cell 128, 1089-1103.

394 Bronzi, P., Rosenthal, H., Gessner, J., 2011. Global sturgeon aquaculture production: An overview. Journal of 395 Applied Ichthyology 27, 169-175.

396 Bullard, J.H., Purdom, E., Hansen, K.D., Dudoit, S., 2010. Evaluation of statistical methods for normalization 397 and differential expression in mRNA-Seq experiments. Bmc Bioinformatics 11, 1-13.

398 Castellano, L., Rizzi, E., Krell, J., Cristina, M.D., Galizi, R., Mori, A., Tam, J., Bellis, G.D., Stebbing, J., 399 Crisanti, A., 2015. The germline of the malaria mosquito produces abundant miRNAs, endo-siRNAs, piRNAs 400 and 29-nt small RNAs. Bmc Genomics 16, 100.

401 Devlin, R.H., Nagahama, Y., 2002. Sex determination and sex differentiation in fish: an overview of genetic, 402 physiological, and environmental influences. Aquaculture 208, 191-364.

403 Dongol, Y., 2011. A Policy Gap Analysis of Convention on International Trade in Endangered Species of Wild 404 Fauna and Flora (CITES) Implementation in Nepal.

405 Falahatkar, B., Falahatkar, S., Gilani, M.H.T., Abbasalizadeh, A., 2011. Laparoscopy, a minimally-invasive 406 technique for sex identification in cultured great sturgeon Huso huso. Aquaculture 321, 273-279.

407 Fontana, F., Congiu, L., Mudrak, V.A., Quattro, J.M., Smith, T.I., Ware, K., Doroshov, S.I., 2008. Evidence of 408 hexaploid karyotype in shortnose sturgeon. 51, 113-119.

409 Girard, A., Sachidanandam, R., Hannon, G.J., Carmell, M.A., 2006. A germline-specific class of small RNAs 410 binds mammalian Piwi proteins. Nature 442, 199-202. 
411 Git A., Dvinge H., Salmon-Divon M., Osborne M., Kutter C., Hadfield J., Bertone P., Caldas C., 2010. 412 Systematic comparison of microarray profiling, real-time PCR, and next-generation sequencing technologies 413 for measuring differential microRNA expression. Rna 16, 991-1006.

414 Ha, H., Song, J., Wang, S., Kapusta, A., Feschotte, C., Chen, K.C., Xing, J., 2014. A comprehensive analysis 415 of piRNAs from adult human testis and their relationship with genes and mobile elements. Bmc Genomics 15 , 416545.

417 Honkala, I., Lobstein, A., 2010. Identification of Acipenseriformes species in trade. Journal of Applied 418 Ichthyology 24, 2-19.

419 Houwing, S., Berezikov, E., Ketting, R.F., 2008. Zili is required for germ cell differentiation and meiosis in 420 zebrafish. Embo Journal 27, 2702-2711.

421 Houwing, S., Kamminga, L.M., Berezikov, E., Cronembold, D., Girard, A., Van, d.E.H., Filippov, D.V., 422 Blaser, H., Raz, E., Moens, C.B., 2007. A role for Piwi and piRNAs in germ cell maintenance and transposon 423 silencing in Zebrafish. Cell 129, 69-82.

424 Jin, S.B., Zhang, Y., Dong, X.L., Xi, Q.K., Song, D., Fu, H.T., Sun, D.J., 2015. Comparative transcriptome 425 analysis of testes and ovaries for the discovery of novel genes from Amur sturgeon (Acipenser schrenckii). 426 Genetics \& Molecular Research Gmr 14, 18913.

427 Juliano, C., Wang, J., Lin, H., 2011. Uniting germline and stem cells: the function of Piwi proteins and the 428 piRNA pathway in diverse organisms. Annual Review of Genetics 45, 447.

429 Kanehisa M, Goto S, Hattori M, Aoki-Kinoshita KF, Itoh M, Kawashima S, Katayama T, Araki M, and 430 Hirakawa M, 2006. From genomics to chemical genomics: new developments in KEGG. Nucleic Acids Res 34, 431 D354-357.

432 Krykhtin, M.L., Svirskii, V.G., 1997. Endemic sturgeons of the Amur River: kaluga, Huso dauricus, and Amur 433 sturgeon, Acipenser schrenckii. Environmental Biology of Fishes 48, 231-239.

434 Langmead, B., Pop, M., 2009. Ultrafast and memory-efficient alignment of short DNA sequences to the human 435 genome. Genome Biology 10, R25.

436 Lau, N.C., Ohsumi, T., Borowsky, M., Kingston, R.E., Blower, M.D., 2009. Systematic and single cell 437 analysis of Xenopus Piwi-interacting RNAs and Xiwi. Embo Journal 28, 2945-2958.

438 Leary, S., Underwood, W., Anthony, R., Cartner, S., Corey, D., Grandin, T., Greenacre, C.B., Gwaltney-Bran, 439 S., Mccrackin, M.A., Meyer, R., 2013. AVMA Guidelines for the Euthanasia of Animals: 2013 Edition. 440 University of Alaska Anchorage.

441 Li, D., Liu, Z., Xie, C., 2012. Effect of stocking density on growth and serum concentrations of thyroid 442 hormones and cortisol in Amur sturgeon, Acipenser schrenckii. Fish Physiology \& Biochemistry 38, 511-520.

443 Li, X.Z., Roy, C., Dong, X., Bolcun-Filas, E., Wang, J., Han, B., Xu, J., Moore, M., Schimenti, J., Weng, Z., 
444 2013. An Ancient Transcription Factor Initiates the Burst of piRNA Production during Early Meiosis in Mouse 445 Testes. Molecular Cell 50, 67-81.

446 Ludwig, A., Belfiore, N.M., Pitra, C., Svirsky, V., Jenneckens, I., 2001. Genome duplication events and 447 functional reduction of ploidy levels in sturgeon (Acipenser, Huso and Scaphirhynchus). Genetics 158, 1203.

448 Malone, C.D., Hannon, G.J., 2009. Small RNAs as Guardians of the Genome. Cell 136, 656-668.

449 Masoudifard, M., Vajhi, A.R., Moghim, M., Nazari, R.M., Naghavi, A.R., Sohrabnejad, M., 2011. High 450 validity sex determination of three years old cultured beluga sturgeon (Huso huso) using ultrasonography. 451 Journal of Applied Ichthyology 27, 643-647.

452 Petochi, B.T., Di, M.P., Donadelli, V., Longobardi, A., Corsalini, I., Bertotto, D., Finoia, M.G., Marino, G., 453 2011. Sex and reproductive stage identification of sturgeon hybrids (Acipenser naccarii $\times$ Acipenser baerii) 454 using different tools: ultrasounds, histology and sex steroids. Journal of Applied Ichthyology 27, 637-642.

455 Pikitch, E.K., Doukakis, P., Lauck, L., Chakrabarty, P., Erickson, D.L., 2005. Status, trends and management 456 of sturgeon and paddlefish fisheries. Fish and Fisheries 6, 233-265.

457 Qingling Yang, J.H., Liu Wang, Bo Xu, Huan Zhang, Nan Ye, Zhiqiang Zhang, Dexin Yu, Howard J. Cooke, 458 Yuanwei Zhang, Qinghua Shi, 2013. MicroRNA and piRNA profiles in normal human testis detected by next 459 generation sequencing. PLoS One 8, e66809.

460 Raymakers, C., Hoover, C., 2010. Acipenseriformes: CITES implementation from Range States to consumer 461 countries. Journal of Applied Ichthyology 18, 629-638.

462 Roovers, E., Rosenkranz, D., Mahdipour, M., Han, C.T., He, N., Chuvadesousalopes, S., Vanderwesterlaken, 463 L.J., Zischler, H., Butter, F., Roelen, B.J., 2015. Piwi Proteins and piRNAs in Mammalian Oocytes and Early 464 Embryos. Cell Reports 10, 2069-2082.

465 Ross, R.J., Weiner, M.M., Lin, H., 2014. PIWI proteins and PIWI-interacting RNAs in the soma. Nature 505, $466 \quad 353-359$.

467 Ruban, G.Q., W., 2013. Acipenser schrenckii. In: IUCN Red List of Threatened Species. Version 2013.2. 468 www.iucnredlist.org.

469 Rojas-Ríos, P., Simonelig, M, 2018. piRNAs and PIWI proteins: regulators of gene expression in development 470 and stem cells. Development 145: dev161786. doi: 10.1242/dev.161786.

471 Vagin VV, S.A., Li C, Seitz H, Gvozdev V, Zamore PD, 2006. A distinct small RNA pathway silences selfish 472 genetic elements in the germline. Science 313, 320-324.

473 Wei, Q.W., Zou, Y., Li, P., Li, L., 2011. Sturgeon aquaculture in China: progress, strategies and prospects 474 assessed on the basis of nation-wide surveys (2007-2009). Journal of Applied Ichthyology 27, 162-168. 
475 Wilczynska, A., Minshall, N., Armisen, J., Miska, E.A., Standart, N., 2009. Two Piwi proteins, Xiwi and Xili, 476 are expressed in the Xenopus female germline. Rna-a Publication of the Rna Society 15, 337-345.

477 Williams, Z., Morozov, P., Mihailovic, A., Lin, C., Puvvula, P.K., Juranek, S., Rosenwaks, Z., Tuschl, T., 478 2015. Discovery and Characterization of piRNAs in the Human Fetal Ovary. Cell Reports 13, 854-863.

479 Yang Q, H.J., Wang L, Xu B, Zhang H, Ye N, Zhang Z, Yu D, Cooke HJ, Zhang Y, Shi Q, 2013. MicroRNA 480 and piRNA Profiles in Normal Human Testis Detected by Next Generation Sequencing. PLoS One 8, e66809.

481 Yuan, L., Zhang, X., Li, L., Jiang, H., Chen, J., 2014. High-Throughput Sequencing of MicroRNA 482 Transcriptome and Expression Assay in the Sturgeon, Acipenser schrenckii. Plos One 9, e115251.

483 Yuan, T., Huang, X., Woodcock, M., Du, M., Dittmar, R., Wang, Y., Tsai, S., Kohli, M., Boardman, L., Patel, 484 T., 2016. Plasma extracellular RNA profiles in healthy and cancer patients. Scientific Reports 6, 19413.

485 Zdobnov EM, Apweiler R, 2001. InterProScan - an integration platform for the signature-recognition methods 486 in InterPro. Bioinformatics 17, 847-848.

487 Zhang, X., Yuan, L., Li, L., Jiang, H., Chen, J., 2016a. Conservation, sex-biased expression and functional 488 annotation of microRNAs in the gonad of Amur sturgeon (Acipenser schrenckii). Comparative Biochemistry 489 \& Physiology Part D Genomics \& Proteomics 18, 54-61.

490 Zhang, X.J., Jiang, H.Y., Li, L.M., Yuan, L.H., Chen, J.P., 2016b. Transcriptome analysis and de novo 491 annotation of the critically endangered Amur sturgeon (Acipenser schrenckii). Genetics \& Molecular Research 49215.

493 Zhang, Y., Wang, X., Kang, L., 2011. A k-mer scheme to predict piRNAs and characterize locust piRNAs. 494 Bioinformatics 27, 771.

495 Zhang, X., Li, L., Jiang, H., Ma, J., Li, J., Chen, J., 2018. Identification and differential expression of 496 microRNAs in testis and ovary of Amur sturgeon (Acipenser schrenckii). Gene 658, 36-46.

497

498

499 ACCESSION CODES 
500 All small RNA data series have been submitted to the SRA database under accession numbers SRR3180645,

501 SRR3180649, SRR3180651, and SRR3180713. The microarray data have been deposited in the ArrayExpress,

502 GEO database under accession number GSE83840.

503

504

505 Tables

506 Table 1. Small RNA annotation of Acipenser schrenckii.

509 Figures

510 Figure 1. Summary of 875,679 sturgeon putative piRNAs. (A) Base utility analysis of putative

511 piRNAs. Black arrow indicates the preference of $5^{\text {' } U}$ and an adenine at position $10^{\text {th }}$. (B)

512 Graphical representation of putative piRNAs between ovary and testis libraries. (C) Pie chart 513 summary of putative piRNA distribution.

515 Figure 2. Base bias analysis of $A$. schrenckii small RNAs. (A) Length distribution of small

516 RNAs reads from somatic tissues, testes and ovaries of Acipenser schrenckii. (B) - (D) Analysis 
517 of the 5' position nucleotide utility of putative miRNA reads and piRNA reads in somatic tissues,

518 testes and ovaries of A. schrenckii. Somatic tissues: data from our previous small RNA

519 transcriptome from a pool of five tissues (brain, heart, muscle, liver and spleen) of A. schrenckii

520 (Yuan et al., 2014) was used as a control.

521

522 Figure 3. Gene ontology (GO) classification annotated for piRNA-generating genes related

523 to reproduction. **: Enriched GO terms related to reproduction in both levels of sex-specific

524 expressed putative piRNAs and total putative piRNAs. Details about enriched GO terms were

525 listed in Table S3 (at total putative piRNAs level) and Table S4 (at sex-specific putative piRNAs 526 level).

527

528 Supplementary Information

529 Figure S1 - The sequence length distribution and frequency percentages of small RNA

530 reads of Acipenser schrenckii. The x-axis indicates the lengths of small RNA reads. The y-axis

531 indicates the percentages of small RNA reads with a specific length. Different colors suggest

532 different types of small RNAs. A) and B), Ovary 1 and 2; C) and D), Testis 1 and 2.

533 Figure S2. Expression pattern of sex-biased putative piRNAs validated by microarray. The

534 expression of 311 sex-biased putative piRNAs is reflected as Log2FC. The heat map showed the 
535 putative piRNAs clustered into ten clades based on sexes, including five clades up-regulated in 536 ovaries and five up-regulated in testes. For detailed information see Table S5.

537 Table S1- Summary of library quality control and the ambiguous reads filter.

538 Table S2 - List of orthologs to known piRNAs.

539 Table S3 - Summary of 264 enriched GO annotations and 29 enriched KEGG pathways

540 that may associate with sturgeon putative piRNA. *: Enriched GO/KEGG terms may involve

541 in reproduction and aquaculture.

542 Table S4 - Summary of $\mathbf{1 3 0}$ enriched GO annotations and 2 enriched KEGG pathways that

543 may associate with sex-specific putative piRNA. *: Enriched GO terms may involve in

544 reproduction and aquaculture.

545 Table S5 - Validation of the expression of 311 sex-biased putative piRNAs by miccroarray. 
Figure 1

Summary of 875,679 sturgeon putative piRNAs.

(A) Base utility analysis of putative piRNAs. Black arrow indicates the preference of 5'U and an adenine at position 10th. (B) Graphical representation of putative piRNAs between ovary and testis libraries. (C) Pie chart summary of putative piRNA distribution.

$\mathbf{A}$

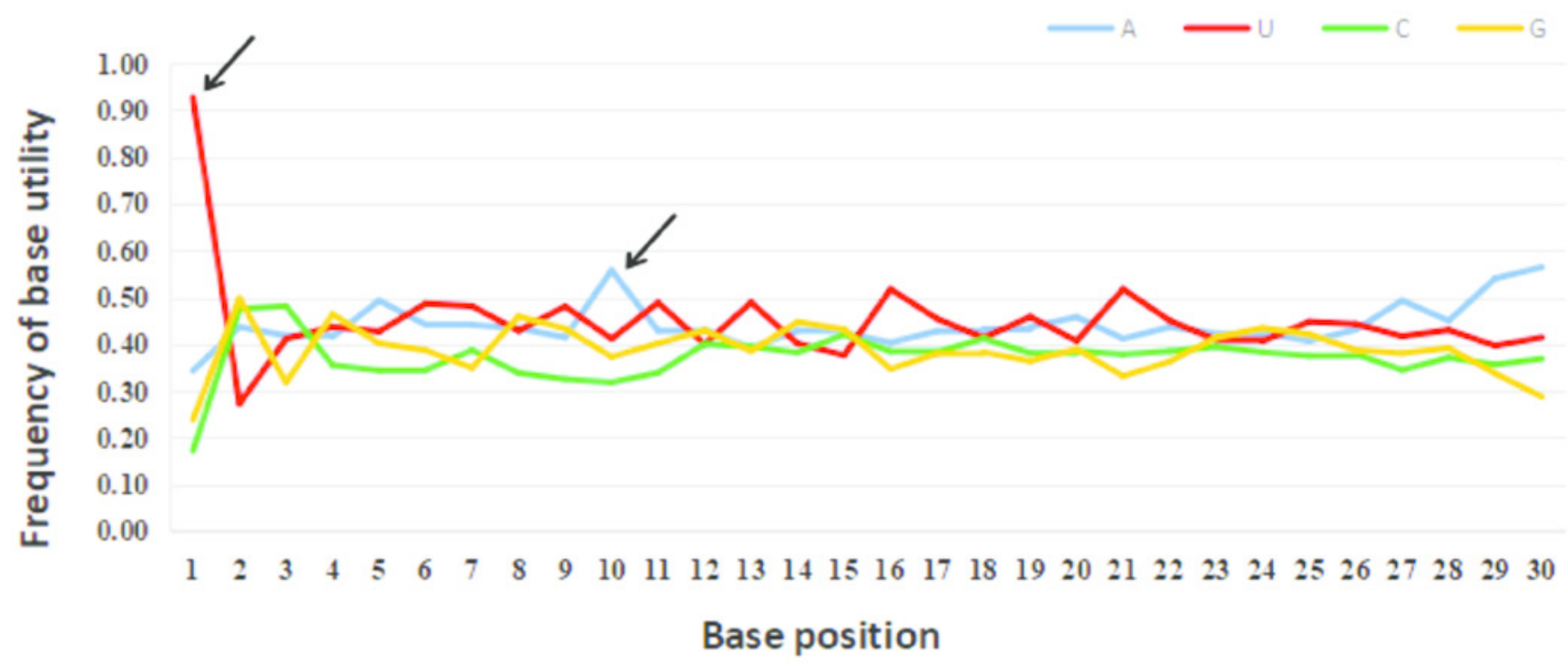

B

C
Ovary
Testis

piRNA distribution on genes
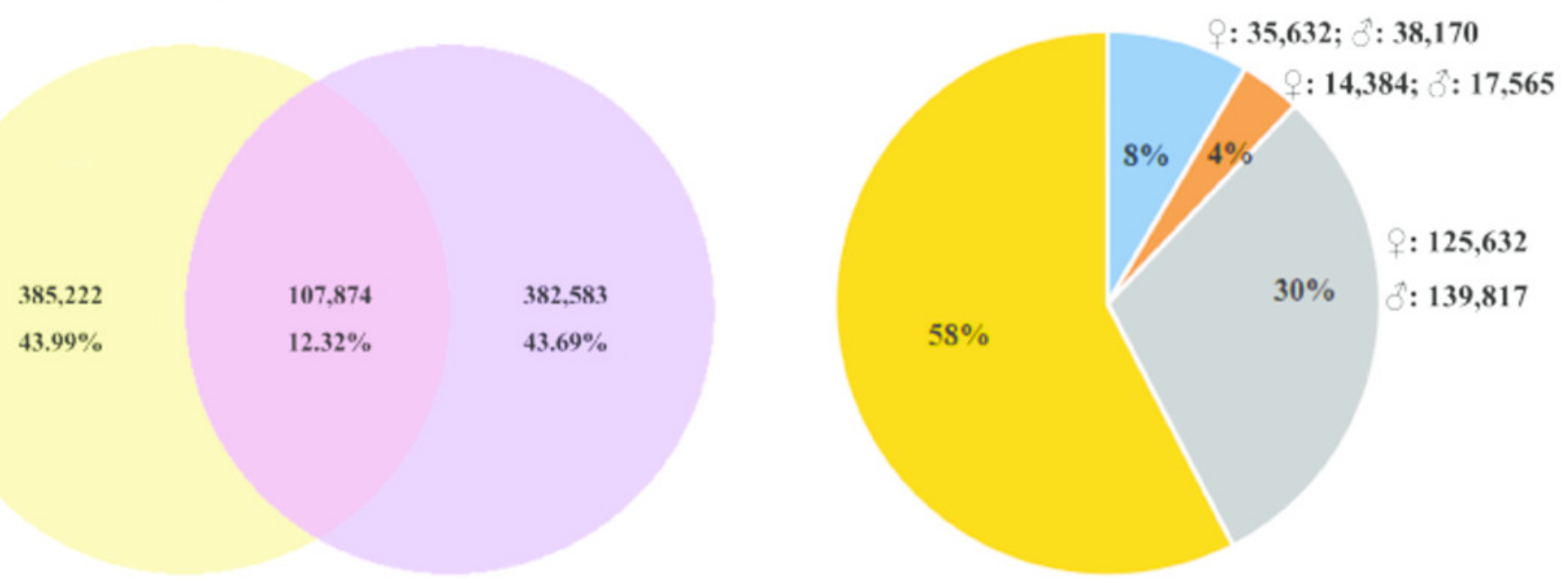
Figure 2

Base bias analysis of A. schrenckii small RNAs.

(A) Length distribution of small RNAs reads from somatic tissues, testes and ovaries of Acipenser schrenckii. (B) - (D) Analysis of the 5' position nucleotide utility of putative miRNA reads and piRNA reads in somatic tissues, testes and ovaries of A. schrenckii. Somatic tissues: data from our previous small RNA transcriptome from a pool of five tissues (brain, heart, muscle, liver and spleen) of A. schrenckii (Yuan et al., 2014) was used as a control.

$\mathbf{A}$

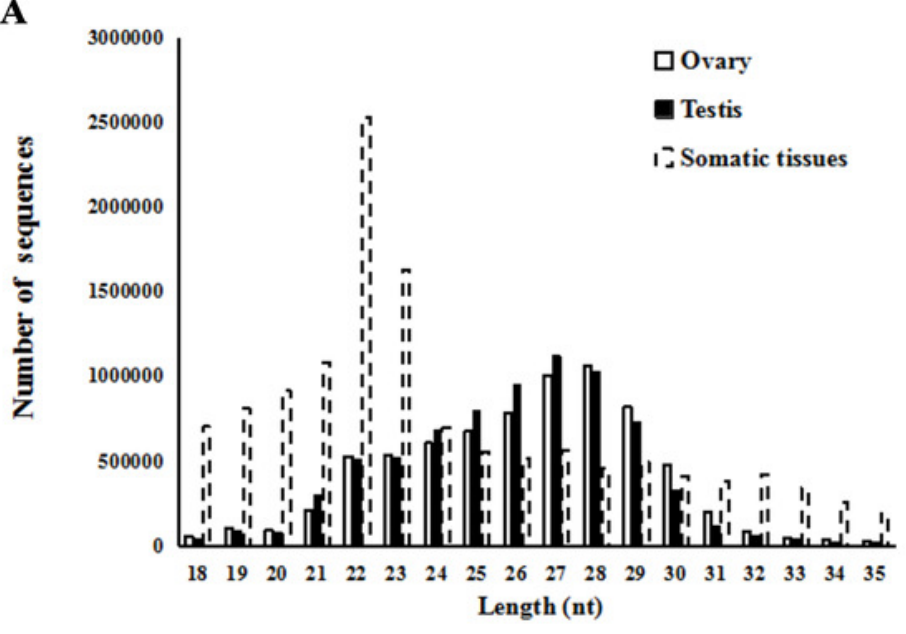

C

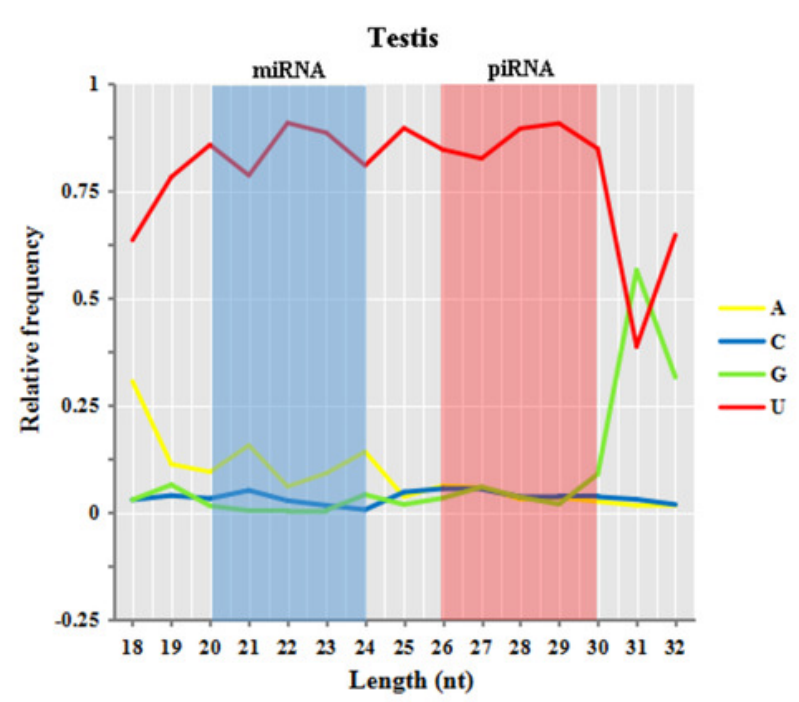

B

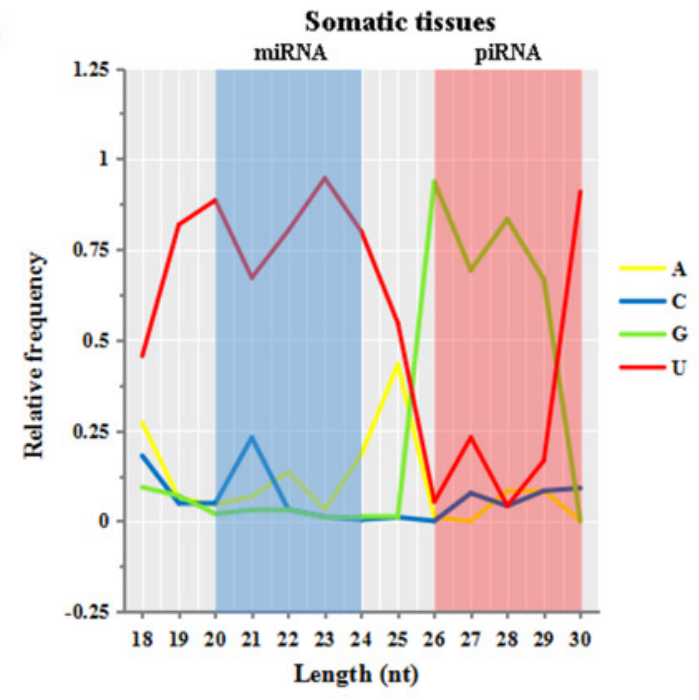

D

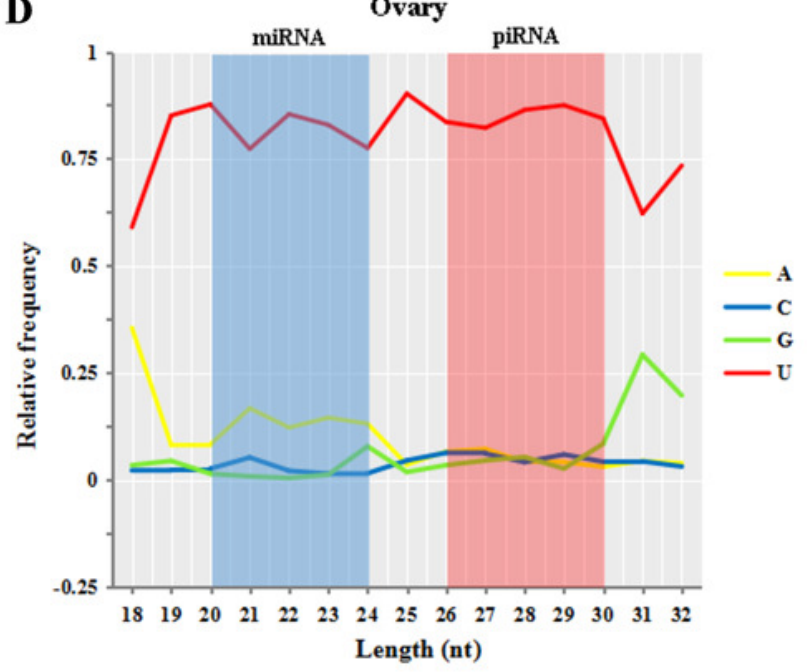


Figure 3

Gene ontology (GO) classification annotated for piRNA-generating genes related to reproduction.

**: Enriched GO terms related to reproduction in both levels of sex-specific expressed putative piRNAs and total putative piRNAs. Details about enriched GO terms were listed in Table S3 (at total putative piRNAs level) and Table S4 (at sex-specific putative piRNAs level).

Enriched Go Terms--BP

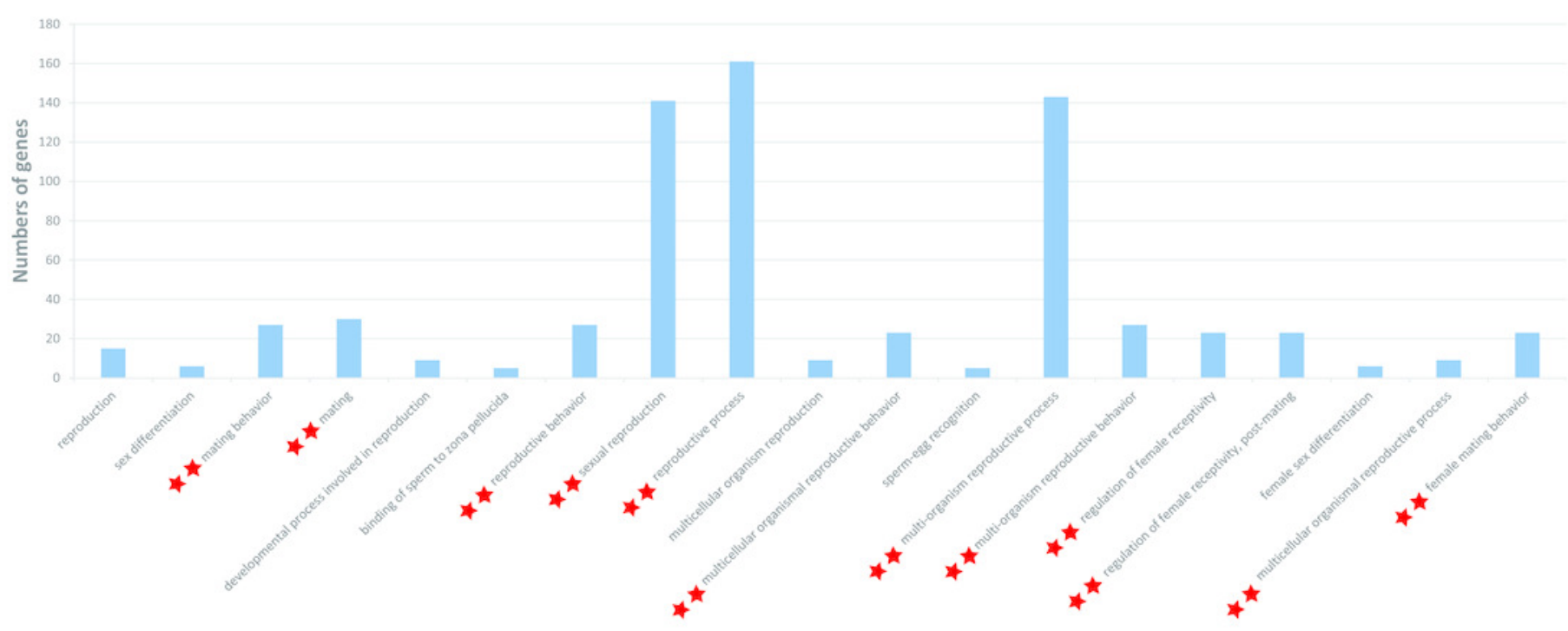




\section{Table $\mathbf{1}$ (on next page)}

Small RNA annotation of Acipenser schrenckii. 
1 Table 1. Small RNA annotation of Acipenser schrenckii.

\begin{tabular}{|c|c|c|c|c|c|c|c|c|c|c|c|}
\hline \multirow{3}{*}{ description } & Mapped & conserved_ & rRNA & tRNA & snRNA & snoRNA & repeats & novel_m & piRNA & uniq & other \\
\hline & sRNA & miRNA & & & & & & iRNA & & piRNA & \\
\hline & 3367185 & 35186 & 25313 & 1 & 21004 & 862 & 10175 & 6055 & 566402 & 272963 & 2704564 \\
\hline \multirow[t]{2}{*}{ Ovary 1} & $(100.00 \%)$ & $(1.04 \%)$ & $(0.75 \%)$ & $(0.00 \%)$ & $(0.62 \%)$ & $(0.03 \%)$ & $(0.30 \%)$ & $(0.18 \%)$ & $(16.75 \%)$ & $(8.1 \%)$ & $(80.32 \%)$ \\
\hline & 3303306 & 52948 & 28912 & 0 & 3595 & 826 & 10615 & 6401 & 595130 & 279287 & 2604879 \\
\hline \multirow[t]{2}{*}{ Ovary 2} & $(100.00 \%)$ & $(1.60 \%)$ & $(0.88 \%)$ & $(0.00 \%)$ & $(0.11 \%)$ & $(0.03 \%)$ & $(0.32 \%)$ & $(0.19 \%)$ & $(18.02 \%)$ & $(8.45 \%)$ & $(78.86 \%)$ \\
\hline & 3164300 & 39703 & 44586 & 1 & 4478 & 641 & 10560 & 6552 & 613914 & 271914 & 2443865 \\
\hline Testis 1 & $(100.00 \%)$ & $(1.25 \%)$ & $(1.41 \%)$ & $(0.00 \%)$ & $(0.14 \%)$ & $(0.02 \%)$ & $(0.33 \%)$ & $(0.21 \%)$ & $(19.40 \%)$ & $(8.59 \%)$ & $(77.23 \%)$ \\
\hline Testis 2 & 3126400 & 67386 & 39749 & 0 & 6539 & 578 & 11264 & 5340 & 620324 & 287659 & 2375220 \\
\hline
\end{tabular}




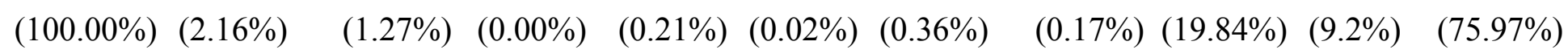

